\title{
A metaheuristic algorithm for the multi-depot vehicle routing problem with heterogeneous fleet
}

\author{
Rubén Iván Bolaños $^{a}$, John Willmer Escobar ${ }^{b^{*}}$ and Mauricio Granada Echeverric
}

${ }^{a}$ Faculty of Engineering, Universidad Tecnológica de Pereira / Integra S.A, Colombia

${ }^{b}$ Department of Accounting and Finance, Universidad del Valle, Colombia

${ }^{c}$ Faculty of Engineering, Universidad Tecnológica de Pereira, Colombia

\begin{tabular}{|c|c|}
\hline$\overline{C H}$ R O N I C LE & A B S T RACT \\
\hline $\begin{array}{l}\text { Article history: } \\
\text { Received August } 182017 \\
\text { Received in Revised Format } \\
\text { August } 252017 \\
\text { Accepted November } 152017 \\
\text { Available online } \\
\text { November } 152017 \\
\text { Keywords: } \\
\text { Heterogeneous fleet } \\
\text { Multi-depot } \\
\text { Vehicle routing problem } \\
\text { Metaheuristics }\end{array}$ & $\begin{array}{l}\text { This paper proposes a metaheuristic algorithm to solve the Multi-Depot Vehicle Routing } \\
\text { Problem with a Heterogeneous Fleet (MDHFVRP). The problem consists of determining the } \\
\text { customers and the vehicles to be assigned to each used depot and the routes to be performed to } \\
\text { fulfill the demands of a set of customers. The objective is to minimize the sum of the fixed cost } \\
\text { associated with the used vehicles and of the variable traveling costs related to the performed } \\
\text { routes. The proposed approach is based on a modified genetic algorithm, which generates an } \\
\text { initial population with heuristic solutions obtained from the well-known (LKH) heuristic } \\
\text { algorithm for the TSP together with the solution of a mathematical model for the shortest path } \\
\text { problem. In addition, two recombination methods and a mutation operator are considered. } \\
\text { Computational experiments on benchmark instances show that the proposed algorithm can } \\
\text { obtain high-quality solutions within short computing times. }\end{array}$ \\
\hline
\end{tabular}

\section{Introduction}

The Vehicle Routing Problem (VRP) is a classic combinatorial optimization problem of great scientific interest in the field of Operations Research due to its high economic impact and its difficulty to be solved. The VRP plays an important role in supply chain optimization for companies whose activities involve the transportation of goods or people. Realistic situations have been the main sources of motivation for the study of new variants of VRP for satisfying the requirements of customers, such as time windows, pickup and delivery, or real aspects of such problems such as route length, multiple depots, heterogeneous fleets, and fixed costs. However, the main objective is focused on the optimization of the transportation resources and the satisfaction of customer demand, independently of the variant of the problem (Toth \& Vigo, 2002).

During the last 5 decades, researchers have proposed new formulations to solve several variants of the Vehicle Routing Problem (VRP), therein incorporating features that describe realistic problems that can be solved easily by exact approaches. Therefore, the scientific community of operations research has

* Corresponding author

E-mail: john.wilmer.escobar@correounivalle.edu.co (J. W. Escobar)

2018 Growing Science Ltd.

doi: $10.5267 / j . j$ ijiec.2017.11.005 
experienced significant growth due to the development of computer systems, which have facilitated great progress in the implementation of powerful, precise and fast algorithms.

This paper considers a variant of the VRP by including multiple depots and a heterogeneous fleet with variable and fixed costs called the Multi-Depot Vehicle Routing Problem with a Heterogeneous Fleet (MDHFVRP). The MDHFVRP is NP-hard because it generalizes two well-known NP-hard problems: the Multi-Depot Vehicle Routing Problem (MDVRP) and the Vehicle Routing Problem with a Heterogeneous Fleet (HFVRP). The MDVRP can be described as a MDHFVRP with a homogeneous fleet, and the HFVRP can be described as a MDHFVRP with only one depot.

\section{Review of the literature}

Although the vehicle routing problem has been widely studied since the first formulation was applied to fuel distribution by Dantzig and Ramser (1959), the version involving multiple deposits and different types of vehicles has not been studied, sufficiently. Early algorithms for the MDHFVRP were proposed by Cassidy and Bennett (1972). This work considers an iterative algorithm that seeks progressive improvements from random or predetermined initial solutions for the scheduling problem of vehicle routing applied to the delivery of school meals. The formulation of this problem considers multiple depots, a heterogeneous fleet of vehicles and their main constraints such as the size and duration of each route. The main objective is to minimize the travel time. The instances used are real and involve 600 schools (customers), 300 training centers and 100 vehicles.

Wren and Holliday (1972) proposed an iterative algorithm for the vehicle routing problem for fulfilling the demand of a set of customers from several depots. The initial solution is generated by a heuristic algorithm based on the well-known procedure proposed by Clarke and Wright (1964), which is applied to each depot, separately. This problem considers a homogeneous and limited fleet for the supply the demand of customers according to their priorities. The obtained results are compared with existing solutions for a single depot.

Renaud et al. (1996) proposed a metaheuristic algorithm based on Tabu Search for solving the problem of supply products to a set of customers from several depots by considering capacity constraints and the size of the routes. The initial solution is generated by the assignment of the customers to the closest depot. The improvement stages consider local search procedures called 1-route, 2-routes and 3-routes. The proposed approach is tested on 23 benchmark instances taken from the literature.

A multilevel heuristic for the MDHFVRP is introduced in Salhi and Sari (1997). The heuristic considers two phases. The first phase uses heuristic called borderline customers to build an initial feasible solution. In the second phase, a local search strategy conducted by improving the initial solution is proposed to avoid a local optima being reached. The proposed heuristic was tested on benchmark instances by considering up to 360 customers, from 2 to 9 depots and 5 different vehicle capacities.

Nagy and Salhi (2005) proposed different heuristic algorithms for the vehicle routing problem with pickup and delivery. The proposed approach can solve the problem by considering one and several depots. The authors used the concept of insertion between routes and allow for integration between the process of pick-up and delivery, simultaneously. A combined methodology for a heuristic algorithm based on the sweep method and a linear integer programming model (Set Partitioning Model) for solving the multidepot vehicle routing problem by considering intermediate depots was proposed by Crevier et al. (2007). An algorithm based on the adaptive memory of a Tabu Search implemented the improvement stage of the proposed approach. Computational experiments were performed on a random set of instances.

A genetic algorithm for solving the Multi-Depot Vehicle Routing Problem was introduced by Ho et al. (2008). The first part of the algorithm considers random initial solutions, while the second phase 
integrates the nearest neighbor heuristic and the saving algorithm to find the initial population. Computational experiments were performed on instances of different sizes. The obtained results demonstrate a better performance under the proposed algorithm by combining different heuristics in the first stage.

Mirabi et al. (2010) developed hybrid heuristics for the MDVRP. The proposed methods combine deterministic, stochastic and Simulated Annealing (SA) heuristics. The objective function is to minimize the delivery time of each vehicle. Computational experiments on a set of randomly generated sets with different sizes of customers and depots were considered. The obtained results demonstrated the efficiency of the proposed methodology.

An adaptation of the MDVRP to the design of an urban fiber optic network for telephony was presented by Baldacci and Dell'Amico (2010). The problem was addressed from graph theory, and different heuristic algorithms were implemented as solution methods. The methodology was tested on a real set of instances and on a set of instances proposed in the literature.

Aras et al. (2011) presented an extension of MDVRP by considering a gross profit and a purchase price for each customer visited. Two mixed integer linear programming models were formulated. A Tabu Search strategy was proposed to solve instances with medium and large sizes. The Tabu Search has a runtime that is slightly better than the ones obtained by exact models solved using a commercial solver.

An integer programming model was presented by Gulczynski et al. (2011) to solve the MDVRP with split deliveries. The objective function was to minimize the distance traveled by the vehicles, by considering either a single depot or multiple depots. The results were obtained on a set of 30 instances. Additionally, a new set of instances of high quality was created.

A genetic clustering algorithm based on a geometric shape was presented by Yücenur and Demirel (2011). The method seeks to assign customers to each depot, minimizing the total distance. The proposed algorithm achieves a better performance against the nearest neighbor heuristic in terms of required computing time.

A related work to the MDHFVRP was proposed by Xu et al. (2012). That paper proposed a new mathematical formulation for the Multi-Depot Vehicle Routing Problem with Heterogeneous Fleet with Time Windows (MDHVRPTW). The solution method involves a modified algorithm based on variable neighborhood search with insertion and swap moves, therein seeking a balance between the quality of the solutions and computing time. The proposed algorithm was tested on benchmarking instances, demonstrating the efficiency of the former methodology.

Kuo and Wang (2012) proposed a local search strategy to solve a variant of the MDVRP by considering freight costs. The solution strategy considers three stages: the first step considers a stochastic method for generating initial solutions, the second step considers four operators selected randomly to find neighboring solutions, and finally, in the third stage, a similar method as the Simulated Annealing heuristic is used to select neighboring shortlisted solutions. The results show the efficiency and effectiveness of the algorithm for solving this variant of the MDVRP.

A new variant of the problem considering the location of the multiple depots (LRP) was considered by Wu et al. (2002), Escobar et al. (2013), and Escobar et al. (2014). Wu et al. (2002) proposed a sequential and iterative method with a Simulated Annealing algorithm as the solution method by considering a homogeneous and unlimited vehicle fleet. The obtained solutions were of good quality, and their algorithms were executed in reasonable computing times. 
A two-phase hybrid heuristic algorithm for solving the LRP that, following a construction phase (first phase), applies a granular Tabu Search with different diversification strategies within the improvement phase (second phase) was proposed by Escobar et al. (2013). In addition, a random perturbation procedure was considered to ensure the algorithm did not fall into a local optimum. The obtained results on benchmarking instances demonstrated the efficiency of the proposed approach considering computing times and new best-known solutions. In Escobar et al. (2014), a new heuristic algorithm for the LRP, called Granular Variable Tabu Neighborhood Search (GVTNS), was proposed. This algorithm combines a Granular Tabu Search within a Variable Neighborhood Search algorithm to obtain high-quality solutions. Indeed, this algorithm can obtain good solutions within short CPU times and improves several best-known solutions from the literature.

Bettinelli et al. (2011) proposed an exact method based on Branch-Cut-Price for the MDHFVRP with time windows. The proposed methodology is based on three search strategies and one column generation strategy. Recently, similar problems related to the MDHFVRP have been proposed by Mancini (2016), Karakatič and Podgorelec (2015) and Braekers et al. (2016).

Few papers have considered the MDHFVRP with the characteristics considered in this work (Salhi et al., 2014; Vidal et al., 2014). In Salhi et al. (2014), a mathematical formulation for the MDHFVRP was introduced. An efficient Variable Neighborhood Search (VNS), which incorporates different operators and a local search neighborhood, was addressed to solve the considered problem. The former algorithm includes a pre-processing scheme to identify borderline customers and a mechanism to aggregate and disaggregate routes between depots to reduce the number of neighboring solutions. This strategy achieves reduced computing time. The algorithm was tested on 26 instances in the literature and obtained competitive results.

Vidal et al. (2014) proposed a dynamic programming methodology to evaluate neighborhood sequences efficiently with an optimal choice of vehicle movements and depots under different variants of vehicle routing problems. In addition, a strategy called rotation, which allows one to determine the first customer to be visited for each route, was developed. An iterated local search and a genetic algorithm considering the former methodology was proposed to solve the MDHFVRP. Different computational tests demonstrated the remarkable performance of the proposed algorithms on benchmark instances proposed in the literature. Indeed, new solutions for the MDVRP and the MDHFVRP were found.

In this paper, we propose a genetic algorithm to solve the MDHFVRP. The main successful contributions of the proposed methodology are the following:

a) A population algorithm that a modification of the genetic algorithm was presented by Holland (1975). This algorithm could be extended easily to the multi-objective version.

b) A hybrid method adapted to the MDVRP that involves a heuristic process and exact solution of the shortest path problem. Therefore, the initial population has good quality and completely diversity.

c) During the improvement stage, eight inter- and intra-route local search strategies are used, which allows the solution space to be explored to achieve improvements.

d) The flexibility of the proposed methodology allows different variants of the vehicle routing problem, including multiple traveling salesmen, classic VRP and the MDHFVRP, to be solved.

e) The encoding uses a data structure based on the methodology of a splitting of the TSP tours by auxiliary graphs proposed by Prins (2009).

The paper is organized as follows. Section 2 presents the description and the mathematical formulation of the MDHFVRP. Section 3 details the proposed approach. Experimental results on the benchmark instances from the literature are presented in Section 4. Finally, conclusions and future research are given in Section 5. 


\section{Description and mathematical formulation of the MDHFVRP}

The Vehicle Routing Problem (VRP), in its simplest form, seeks to design routes to fulfill the demand of a set of customers who are geographically dispersed from a depot with a set of homogeneous fleets (Toth \& Vigo, 2002). This problem has been extensively studied for more than 50 years; however, today, it is a growing field in operations research due to the economic impact, which represents a real-life application for companies that provide services and/or products. Indeed, real problems in the field of logistics have led researchers develop new variants of the VRP, therein representing new situations in a more precise manner. Several practical applications have led to a variety of attributes that complement the classic example of the VRP, therein approximating the requirements of different customers (schedules and assignments) and the characteristics of the supply network and vehicles (fleet heterogeneity, multiple depots and fixed costs).

According to Salhi et al. (2014), the MDHFVRP can be defined as the following graph theory problem. Let $G=(V, E)$ be a complete undirected graph, where $V=I \cup J=\{1,2, \cdots, n+m\}$ is the vertex set and $E=\{(i, j): i \in V, j \in V\}$ is the edge set. The vertices $I=\{1, \cdots, m\}$ correspond to the depots, and the vertices $J=\{m+1, \cdots, m+n\}$ correspond to the customers, each with a nonnegative demand $q_{j}$. A set of unlimited heterogeneous vehicles $K$, each with capacity $Q_{k}$, is available at each depot $i$. Each vehicle, when used by a depot to perform a single route, incurs a nonnegative fixed cost $F_{k}$ and variable $\operatorname{cost} \alpha_{k}$ per unit of traveled distance. A nonnegative $\operatorname{cost} C_{i j}$ is associated with each edge. The number of available vehicles at each depot is unlimited.

The goal of the MDHFVRP is to determine the customers and the vehicles to be assigned to each depot and the routes to be constructed to fulfill the demands of the customers with the minimum global cost, given by the sum of the fixed costs of the used vehicles and the variable costs per unit of traveled distance (sum of the edges traveled by each route multiplied by the corresponding variable cost $\alpha_{k}$ of the used vehicles). The following constraints are imposed:

a) Each route must start and finish at the same depot;

b) Each customer is visited exactly once by a single route;

c) The sum of the demands of the customers visited by each vehicle $k$ must not exceed the vehicle's corresponding capacity $Q_{k}$;

d) The total duration of each route (given by the sum of the traveling costs of the traversed edges and of the service times of the visited customers) must not exceed a given value $D$ and

e) Connections between depots are not allowed.

The set of variables used in the mathematical formulation proposed by Salhi et al. (2014) are the following:

$X_{i j k d}$ : is a binary variable equal to 1 if the type of vehicle $k$ travels on the edge $(i, j)$ from the depot $d$ and 0 otherwise.

$Y_{i j}$ : is a continuous non-negative variable that indicates the total load of the vehicle before visiting the node $j$ while traveling on the edge $(i, j)$

\subsection{Mathematical model}

$\min Z=\sum_{d=n+1}^{n+m} \sum_{k=1}^{K} F_{k} \sum_{i=n+1}^{n+m} \sum_{j=1}^{n} X_{i j k d}+\sum_{d=n+1}^{n+m} \sum_{k=1}^{K} \sum_{i=1}^{n+m} \sum_{j=1}^{n+m} \alpha_{k} C_{i j} X_{i j k d}$

s.t. 


$$
\begin{aligned}
& \sum_{d=n+1}^{n+m} \sum_{k=1}^{K} \sum_{i=1}^{n+m} X_{i j k d}=1 ; \quad \forall j=1, \ldots, n \\
& \sum_{d=n+1}^{n+m} \sum_{k=1}^{K} \sum_{j=1}^{n+m} X_{i j k d}=1 ; \quad \forall i=1, \ldots, n \\
& \sum^{n+m} X_{i j k d}^{n+m} \sum_{j i k d i} \quad \forall k=1, \ldots, K \text {; } \\
& \sum_{i=1} X_{i j k d}=\sum_{i=1} X_{j i k d} ; \quad \forall j=1, \ldots, n+m ; \\
& \sum_{i=n+1}^{n+m} \sum_{j=1}^{n} Y_{i j}=\sum_{j=1}^{n} q_{j} \\
& \sum_{i=1}^{n+m} Y_{i j}-\sum_{i=1}^{n+m} Y_{j i}=q_{j} \\
& Y_{i j} \leq \sum^{n+m} \sum^{K} Q_{k} X_{i j k d i} \quad \forall i=1, \ldots, n+m \text {; } \\
& Y_{i j} \leq \sum_{d=n+1} \sum_{k=1} Q_{k} X_{i j k d} ; \quad \forall j=1, \ldots, n \\
& X_{d_{1} i k d_{2}}=0 ; \quad k=1, \ldots, K \text {; } \\
& d_{1} \neq d_{2}=n+1, \ldots, n+m \\
& \forall i=1, \ldots, n \text {; } \\
& X_{i d_{1} k d_{2}}=0 \text {; } \\
& \forall k=1, \ldots, K \text {; } \\
& \forall d_{1} \neq d_{2}=n+1, \ldots, n+m \\
& \forall i, j=1, \ldots, n+m \text {; } \\
& X_{i j k d} \in\{0,1\} \\
& \forall k=1, \ldots, K \text {; } \\
& \forall d=n+1, \ldots n+m \\
& Y_{i j} \geq 0 \\
& \forall i, j=1, \ldots, n+m
\end{aligned}
$$

Eq. (1) represents the total cost, which considers the sum of the fixed cost of the used vehicles and the variable cost for the traveled distance. Eq. (2) and Eq. (3) ensure that each customer must be visited only once. Constraints (4) ensure flow conservation. Eq. (5) shows that the sum of the load coming out of all depots is exactly equal to the sum of the demand of all customers, and Eq. (6) ensures that the amount of charge remaining in the vehicle after visiting customer $j$ is equal to the amount of charge before visiting this customer less its demand. Eq. (7) controls the vehicle capacity constraints. Eq. (8) and Eq. (9) impose that each vehicle departing from a given depot must return to said depot. Finally, Eq. (10) represents the set of used binary variables, and Eq. (11) represents the set of non-negative continuous

\subsection{Proposed approach}

This section describes the general framework of the proposed approach for the MDHFVRP. This algorithm is based on a modified genetic algorithm Chu-Beasley (AGCBM). The AGCBM approach considers a diverse population obtained by a hybrid process. The proposed approach includes a step consisting of improvement procedures after applying the genetic operators of selection, recombination and mutation. The proposed algorithm requires a completely diverse initial population obtained by a hybrid procedure. This procedure considers a unique set of chromosomes by considering only the set of customers. Then, a selection process is performed via two tournaments, each with two randomly selected individuals from the current population. The individual winners of each tournament are used to develop 
a specially adapted recombination process for the MDHFVRP. The newly obtained solution (offspring) is split into routes using a hybrid process. Later, a mutation procedure that considers several intra-route and inter-route moves is applied. Finally, a new individual is joined to the current population by considering the criteria of diversity and quality. The process is performed iteratively until a given number of iterations is reached.

The following are the most remarkable characteristics of the proposed approach:

a) The former algorithm uses the objective function to identify the value of a solution of better quality and to manage the infeasibility for the replacement process of a solution by another one.

b) The proposed algorithm allows one to generate and replace only one individual in each generation cycle.

c) Each individual belonging to the population must be different from the other individuals, thus avoiding premature convergence of the proposed approach to local optima.

d) We have included an aspiration criterion that allows an individual to belong to the current population, despite it not meeting the diversity criteria, only if the solution of the individual is better than the incumbent solution.

\subsection{Encoding used for the MDHFVRP}

The proposed genetic algorithm features a solution representation scheme for the MDHFVRP. A solution is represented by a data structure containing the following attributes: a vector with a giant TSP including all the customers, a matrix of the resulting routes following the hybrid process, a vector with the type of vehicle assigned to each route, a vector with each depot assigned to each route, a vector with the cost of each route, and a variable with the value of the objective function. The following shows a data structure that represents the encoding for the MDHFVRP from a sequence of TSP of 8 customers.

$$
\text { Sol }=\left\{\begin{array}{l}
\text { seq }=\left[\begin{array}{llllllll}
1 & 2 & 3 & 4 & 5 & 6 & 7 & 8
\end{array}\right] \\
R=\left[\begin{array}{lll}
1 & 2 & \\
3 & 4 & 5 \\
6 & & \\
7 & 8 &
\end{array}\right] \\
C=\left[\begin{array}{llll}
c_{1} & c_{2} & c_{3} & c_{4}
\end{array}\right] \\
\text { Dep }=\left[\begin{array}{llll}
d_{1} & d_{2} & d_{3} & d_{4}
\end{array}\right] \\
V=\left[\begin{array}{llll}
v_{1} & v_{2} & v_{3} & v_{4}
\end{array}\right] \\
\text { ObjF }
\end{array}\right.
$$

\subsection{Initial Population: hybrid process}

\subsubsection{Sequences of the giant TSP}

The first stage of the construction of the initial population is obtained through the solutions of several Traveling Salesman Problems (TSPs) by considering the following: $a$ ) the complete set of customers without considering the depots, $b$ ) the different starting vertices for each TSP solution, and $c$ ) a vehicle $k$ with unlimited capacity. This procedure allows one to find $N$ sequences of customers called giant TSP 
tours. With each sequence, it is possible to obtain high-quality individuals following a splitting procedure by selecting prominent edges for several multi-depot vehicle routing problems (Escobar et al., 2013, 2014a, 2014b, 2015; Bolaños et al., 2015; Linfati et al., 2015).

The $N$ sequences are constructed by considering the following procedures: A Nearest Neighborhood heuristic (Gutin et al., 2002), a Lin-Kernighan heuristic (Lin \& Kernighan, 1973) and random permutations of customers. Each of these procedures determines the sequence of a TSP, as shown in Fig. 1.

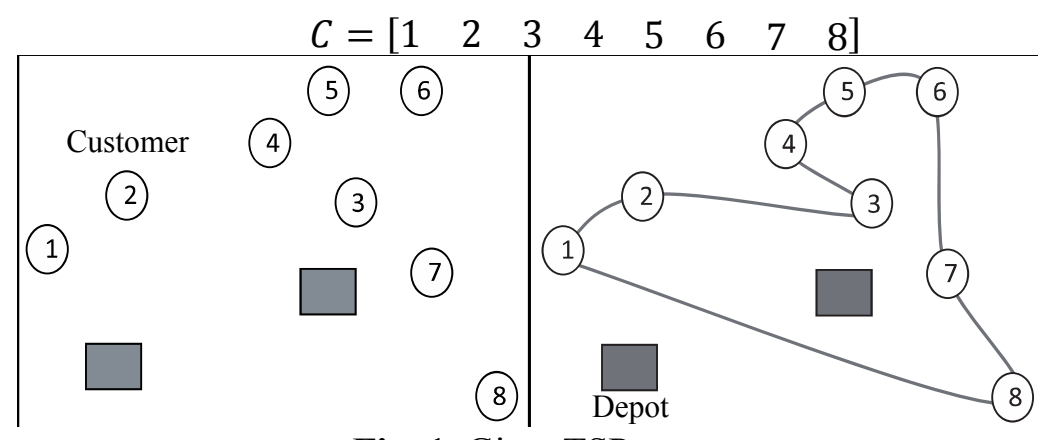

Fig. 1. Giant TSP tour

\subsubsection{Splitting procedure for TSP tours}

The splitting procedure for each TSP is based on the procedure proposed by Prins (2009) for the classical VRP. We have proposed a generalization of this procedure for the MDHFVRP.

Splitting for the VRP

The procedure proposed by Prins (2009) for the VRP performs the following steps:

a) According to the order of attention given by the sequence of a giant TSP, an acyclic graph $H=$ $(X, A)$ is constructed with $n+1$ nodes. In this case, $X$ contains a dummy node 0 plus $n$ vertices representing the set of customers in the order established by each of the sequences $C=\left(C_{1}, \ldots, C_{n}\right)$ The set $A$ is built by adding an $\operatorname{arc}(i-1, j)$ for each subsequence $\left(C_{i}, C_{i+1}, \ldots, C_{j}\right)$ only if the performed route for this sequence is feasible. In other words, if the sum of the demands of the customers belonging to the subsequence $\left(W_{i j}\right)$ must not exceed the vehicle capacity (12), then.

$W_{i j}=\sum_{p=i}^{j} q\left(C_{p}\right) \leq Q$

b) The cost of each subsequence $\left(\mathrm{Z}_{\mathrm{ij}}\right)$ is given by the cost of the traveled $\operatorname{arcs}\left(\mathrm{L}_{\mathrm{ij}}\right)$ which is calculated as follows

$Z_{i j}=L_{i j}=D\left(0, C_{i}\right)+\sum_{p=i}^{j-1} D\left(C_{p}, C_{p+1}\right)+D\left(C_{j}, 0\right)$

c) The splitting procedure of each sequence $C$ is performed by finding the optimal solution for the shortest path between node 0 and node $\mathrm{n}$ of graph $\mathrm{H}$. The mathematical model for the shortest path problem is solved on an auxiliary graph containing all feasible subsequences.

d) The mathematical model for the shortest path problem allows one to find the optimal path between any two vertices of a graph. Let $\mathrm{n}$ be the set of nodes of a graph, and let $\mathrm{s}$ and $\mathrm{t}$ be a pair of nodes for which we want to find the shortest route. We also define $x_{i j}$ as the amount of flow in the arc $(i, j)$, and we define $c_{i j}$ as the cost of the arc $(i, j)$. The mathematical model is described by Eqs. (14-16): 


$$
\begin{aligned}
& \min Z_{2}=\sum_{i=1}^{n} \sum_{j=1}^{n} C_{i j} x_{i j} \\
& \sum_{j=1}^{n} x_{i j}-\sum_{k=1}^{n} x_{k i}=\left\{\begin{array}{rc}
1 & \text { if } i=s \\
-1 & \text { if } i=t \\
0 & \text { otherwise }
\end{array}\right. \\
& x_{i j} \in\{0,1\}
\end{aligned} \quad \forall i=1, \ldots, n
$$

Eq. (14) represents the objective function, given by the sum of the arcs used on the performed path from vertex $s$ to $t$. Eq. (15) ensures flow conservation, and finally, Eq. (16) represents the set of binary variables, which are assigned a value of 1 if the flow between $(i, j)$ is considered on the performed path and 0 otherwise.

Fig. 2 shows an example for the case of the classical VRP in which the giant TSP tour is given by the sequence $C=\left[\begin{array}{lllll}a & b & c & d & e\end{array}\right]$. Note that Fig. 2a shows an acyclic graph $H$, which represents the sequence $C$ along with possible arcs that should depart from and return to the depot. The demand of each customer is given in parentheses, and the travel cost is associated with each arc. Each of the extracted feasible travel sequences $C$ is modeled by an arc on the auxiliary graph in Fig. 2b. The highlighted arcs correspond to the optimal partition, therein applying the hybrid procedure, starting from vertex 0 and finishing with vertex $e$. The vehicle capacity used is equal to 10. Fig. 2c shows the three routes obtained by performing the decoding of each trip at a total cost of 205 (Prins, 2009).

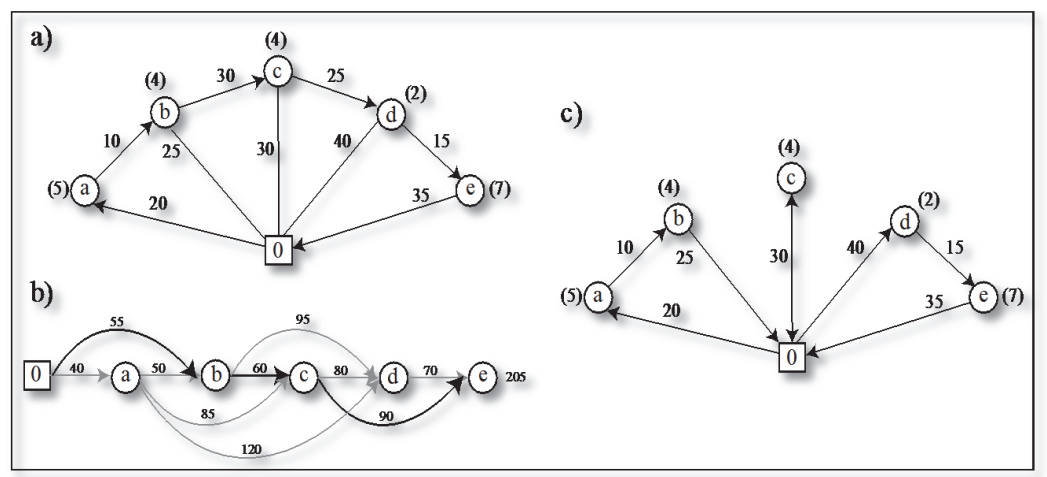

Fig. 2. Splitting procedure applied to the VRP

\subsubsection{Proposed splitting procedure for the MDHFVRP}

According to the order of attention given by the sequence $C$ of a giant TSP, an acyclic graph $H=(X, A)$ is constructed with $n+1$ nodes. However, new conditions must be considered due to the characteristics of the MDHFVRP. A tour is feasible only if the sum of the demands $\left(W_{i j}\right)$ of the customers belonging to the subsequence $\left(C_{i}, C_{i+1}, \ldots, C_{j}\right)$ does not exceed the higher capacity $\left(Q_{k \max }\right)$ of the available vehicles (17).

$$
W_{i j} \leq Q_{k} \max
$$

The cost of each subsequence $\left(Z_{i j k d}\right)$ is obtained by considering both the fixed cost $\left(F_{k}\right)$ and the variable cost $\left(\alpha_{k}\right)$ for each vehicle $k$, with $k=1,2, \ldots, K$, and the selection of the correct depot for each subsequence. The value of the arcs for each subsequence is calculated as follows (18):

$$
L_{i j}=\sum_{p=i}^{j-1} D\left(C_{p}, C_{p+1}\right)
$$


In addition, the assignment of the best vehicle for each subsequence is determined by Eq. (19):

$$
Z_{i j k}=\min \left(F_{k}+\alpha_{k} L_{i j}\right) ; \quad \forall k=1,2, \ldots, K ; \quad W_{i j} \leq Q_{k}
$$

Finally, the depot to be selected for each subsequence is obtained by equation (20):

$$
Z_{i j k d}=Z_{i j k}+\min \left(\alpha_{k}\left[D\left(d, C_{i}\right)+D\left(C_{j}, d\right)\right]\right) ; \quad \forall d=1,2, \ldots, m
$$

Therefore, the total cost for each tour obtained for the graph $\mathrm{H}$ is described by an arc in the auxiliary digraph as follows: $\left[A_{l}=\left(Z_{i j k d}, d, k\right) ; \forall l \mid l=(i, j)\right.$ factible]. Finally, the mathematical model for the shortest path problem is solved on the auxiliary graph containing all feasible subsequences such as the VRP case.

\subsection{Selection}

This process considers the selection of two sequences (individuals) of the initial population to perform two tournaments, each with two randomly selected individuals.

The criterion for choosing the winner of each tournament is based on the value of the objective function associated with each selected sequence. Then, the selection process is illustrated as follow:

\section{Tournament 1}

$$
\begin{aligned}
& S e q_{i}=\left[\begin{array}{llllllll}
1 & 2 & 3 & 4 & 5 & 6 & 7 & 8
\end{array}\right] \\
& \mathrm{Seq}_{j}=\left[\begin{array}{llllllll}
8 & 7 & 6 & 5 & 4 & 3 & 2 & 1
\end{array}\right]
\end{aligned}
$$

\section{Tournament 2}

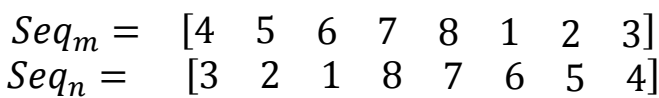

$$
\begin{aligned}
& \text { Winners } \\
& \mathrm{Seq}_{i}=\left[\begin{array}{llllllll}
1 & 2 & 3 & 4 & 5 & 6 & 7 & 8
\end{array}\right] \\
& S e q_{m}=\left[\begin{array}{llllllll}
4 & 5 & 6 & 7 & 8 & 1 & 2 & 3
\end{array}\right]
\end{aligned}
$$

\subsection{Recombination}

The recombination process requires special attention to avoid infeasible solutions because the encoding is based on permutations. One of the constraints of the MDHFVRP is that each customer must be visited once and that the demands of all customers must be satisfied; therefore, two genes within a chromosome should not have the same value. Different algorithm operators, such as PMX (Goldberg \& Lingle, 1985), OBX (Syswerda, 1991), OX and CX (Oliver et al., 1987), can be applied to avoid infeasible sequences. The proposed approach applies the first two operators together because they offer better performance than most other crossover techniques.

\subsection{Recombination PMX}

In the PMX operator, a parent $i$ donates a swath of its genetic part, and the corresponding swath of the parent $j$ is added to the child. Once this process is performed, the remaining alleles are replicated directly from parent $j$. 
In particular, the swath of the genetic part from parent $i$ is randomly selected and copied directly to the child. Then, the remaining positions in the child are filled with the swath genetic information of the parent $j$ by maintaining their order. If there are repeated values, these values are replaced by the information obtained from the same segment positions removed from parent $i$ in parent $j$. The process is performed as follows:

$$
\begin{aligned}
& \multicolumn{10}{c}{\downarrow} \\
& \text {Seq}_{i}=\left[\begin{array}{llllllll}
1 & 2 & 3 & 4 & 5 & 6 & 7 & 8
\end{array}\right] \\
& \text {Seq}_{j}=\left[\begin{array}{lllllllll}
4 & 5 & 6 & 7 & 8 & 1 & 2 & 3
\end{array}\right] \\
& \text { Child }=\left[\begin{array}{llllllll}
4 & 5 & 3 & 4 & 5 & 1 & 2 & 3
\end{array}\right] \rightarrow \text { Infeasible } \\
& \text { Child }=\left[\begin{array}{llllllll}
7 & 8 & 3 & 4 & 5 & 1 & 2 & 6
\end{array}\right] \rightarrow \text { Feasible }
\end{aligned}
$$

\subsection{Recombination $O B X$}

OBX is a slight variation of the PBX operator. We randomly select a set of positions in parent $\mathrm{i}$, which are copied to the child while maintaining their order. Then, the remaining positions are filled with the positions in parent $\mathrm{j}$ (maintaining their sequence) except those in selected positions in parent $\mathrm{i}$. The process is performed as follows:

$$
\begin{aligned}
& \operatorname{Seq}_{i}=\left[\begin{array}{llllllll}
1 & 2 & 3 & 4 & 5 & 6 & 7 & 8
\end{array}\right] \\
& \mathrm{Seq}_{j}=\left[\begin{array}{llllllll}
8 & 7 & 2 & 3 & 6 & 5 & 1 & 4
\end{array}\right] \\
& \text { Child }=\left[\begin{array}{llllllll}
1 & 2 & 3 & 4 & 8 & 7 & 6 & 5
\end{array}\right]
\end{aligned}
$$

Once the recombination procedure is performed, the offspring is split by the hybrid process described in section 3.2.2. Thus, a set of routes is obtained with the additional attributes belonging to the encoding of the MDHFVRP. Later, a mutation procedure is applied.

\subsection{Mutation}

A special mutation process is applied to the obtained offspring. The mutation process is based on the implementation of a process for neighborhood structures that seeks to improve each of the routes obtained by performing a splitting of the offspring. The improvement structures are known as inter- and intraroute local search procedures (Subramanian, Vaz-Penna, Uchoa, \& Ochi, 2012).

\subsection{Inter-route Local Search Procedures}

The inter-route local search procedure starts by considering a list of random routes. Then, six neighborhood structures involving moves between a pair of selected routes are implemented. The solution space of the six improvement strategies is comprehensively explored, i.e., all possible combinations are examined, but only the best moves are performed for each neighborhood structure. The six structures of the inter-neighborhood routes are described below:

a) Shift (1,0): A customer $C_{i}$ belonging to route $R_{k}$ is transferred to route $R_{l}$ The following example shows that customer 6 , which belongs to route 3 , is transferred to the final position on route 2 .

$$
R=\left[\begin{array}{lll}
1 & 2 & \\
3 & 4 & 5 \\
6 & & \\
7 & 8 &
\end{array}\right] \rightarrow R=\left[\begin{array}{llll}
1 & 2 & & \\
3 & 4 & 5 & 6 \\
7 & 8 & &
\end{array}\right]
$$


b) Shift $\left(\mathbf{2 , 0 )}\right.$ : Two consecutive customers $C_{i}$ and $C_{i+1}$ belonging to route $R_{k}$ are transferred to route $R_{1}$. The following example shows that customers 3 and 6 , belonging to the route 2 , are transferred to route 3 .

$$
R=\left[\begin{array}{llll}
1 & 2 & & \\
3 & 6 & 4 & 5 \\
7 & 8 & & \rightarrow
\end{array}\right] \quad R=\left[\begin{array}{llll}
1 & 2 & & \\
4 & 5 & & \\
3 & 6 & 7 & 8
\end{array}\right]
$$

c) Shift $(3,0)$ : Three consecutive customers $C_{i}, C_{i+1}$ and $C_{i+2}$ belonging to route $R_{k}$ are transferred to route $\mathrm{R}_{\mathrm{l}}$. An example of this move is described below.

$$
R=\left[\begin{array}{llll}
1 & 2 & & \\
4 & 5 & & \\
3 & 6 & 7 & 8
\end{array}\right] \rightarrow R=\left[\begin{array}{lllll}
1 & 2 & & & \\
4 & 5 & 3 & 6 & 7 \\
8 & & & &
\end{array}\right]
$$

d) Swap (1,1): Two customers belonging to different routes exchange their positions, i.e., a customer $C_{i}$ belonging to route $R_{k}$ exchanges its position with customer $C_{j}$ of route $R_{l}$. An example is shown as follows:

$$
R=\left[\begin{array}{llll}
1 & 4 & & \\
2 & 5 & & \\
3 & 6 & 7 & 8
\end{array}\right] \rightarrow R=\left[\begin{array}{llll}
1 & 2 & & \\
4 & 5 & & \\
3 & 6 & 7 & 8
\end{array}\right]
$$

e) Swap $(2,1)$ : Two consecutive customers $C_{i}$ and $C_{i+1}$ belonging to route $R_{k}$ are exchanged by a customer $C_{j}$ belonging to route $R_{l}$. The following example shows customers 3 and 6 belonging to route 2 being exchanged by customer 5 of route 3 .

$$
R=\left[\begin{array}{lll}
1 & 2 & \\
3 & 6 & 4 \\
5 & 7 & 8
\end{array}\right] \rightarrow R=\left[\begin{array}{llll}
1 & 2 & & \\
5 & 4 & & \\
3 & 6 & 7 & 8
\end{array}\right]
$$

f) Swap (2,2): This move is an extension of the move Swap $(1,1)$ and considers two pairs of consecutive customers. The edge connecting each pair of customers is kept. The exchange is performed only for a pair of customers belonging to two different routes. An example of the implemented move is described below:

$$
R=\left[\begin{array}{llll}
1 & & & \\
2 & 5 & 7 & 8 \\
3 & 6 & 4 &
\end{array}\right] \rightarrow R=\left[\begin{array}{llll}
1 & & & \\
3 & 6 & 7 & 8 \\
2 & 5 & 4 &
\end{array}\right]
$$

\subsection{Intra-route Local Search Procedures}

Two local search strategies are considered as neighborhood structures:

a) Rotation: This structure considers the rotation of the sequence of the route $R_{k}$ seeking to find a new route whose initial and final customers are close to one of the other depots, i.e., the strategy seeks to find the best sequence for each route and assign it to the best depot. All possible sequences resulting from the rotation process are evaluated. 
The following example shows the rotation procedure applied to route 2 . The results are four new sequences, where the best sequence is $R_{2}=\left[\begin{array}{llll}4 & 5 & 6 & 3\end{array}\right]$. In addition, it is possible to determine the best depot for fulfilling the demand of the new route.

$$
R=\left[\begin{array}{llll}
1 & 2 & & \\
3 & 4 & 5 & 6 \\
7 & 8 & &
\end{array}\right] \rightarrow \begin{array}{llll}
3 & 4 & 5 & 6 \\
4 & 5 & 6 & 3 \\
5 & 6 & 3 & 4 \\
6 & 3 & 4 & 5
\end{array} \text {. }
$$

b) 2-Opt: This is a modified version of the well-known 2-opt move, in which two non-consecutive edges $A(i, i+1)$ and $A(j, j+1)$ are removed and replaced by other edges $A\left(i^{\prime}, i^{\prime}+1\right)$ and $A\left(j^{\prime}, j^{\prime}+\right.$ 1). Fig. 3 shows an example of a 2-Opt move.

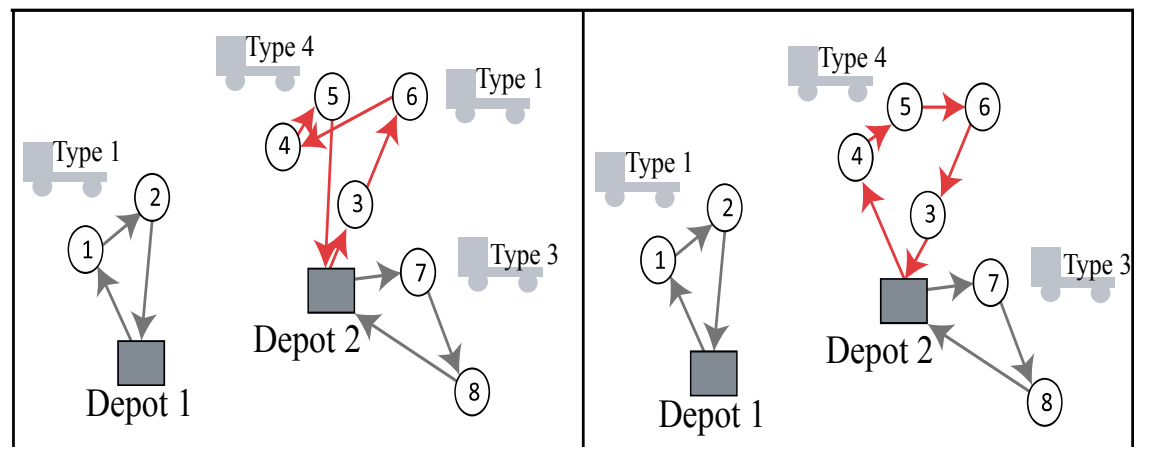

Fig. 3. 2-Opt strategy

\subsection{Joining the population}

When a new individual is obtained by the genetic operators of selection, recombination and mutation, it is necessary to determine whether this new individual could join the current population. The steps for the replacement are the following:

a) Check that the value of the objective function of the new individual is smaller than the value of the objective function of the worst individual belonging to the current population.

b) Check that the giant tour representing the individual does not belong to the current population, i.e., a minimum level of diversity in each sequence of the population is required.

If the individual satisfies both conditions, then it is joined to the population by replacing the individual of poor quality.

\subsection{Pseudocode of the proposed approach}

Algorithm 1 provides an overview of each of the steps of the proposed approach to solving the problem of vehicle routing with multiple deposits and a heterogeneous fleet. 
Algorithm 1: Proposed approach for MDHFVRP

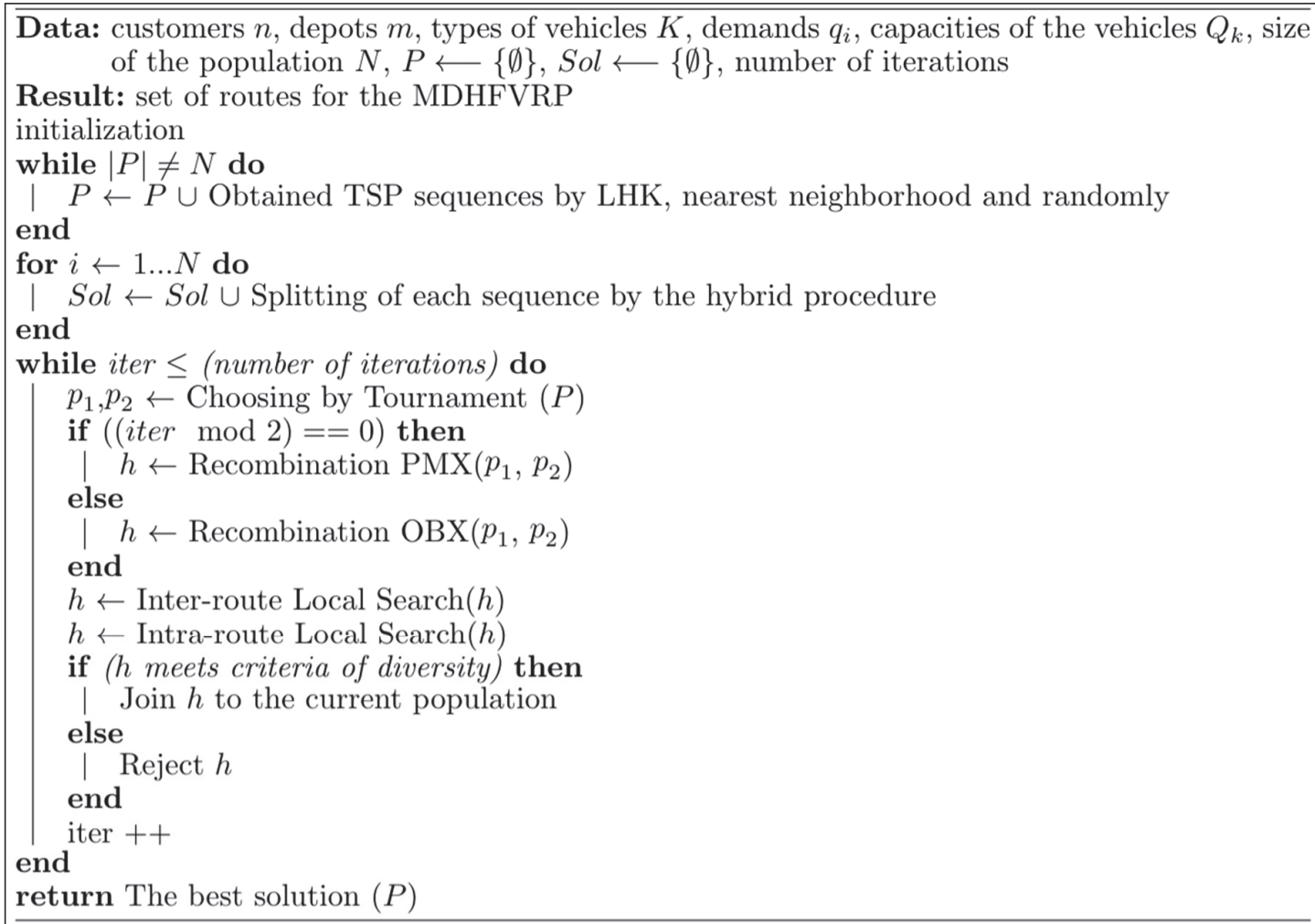

\section{Results}

Several computational experiments have been performed to analyze the behavior and contribution of the proposed approach. The results are compared with existing methods for the MDHFVRP. The tests are performed on the same instances used by Salhi and Sari (1997), Salhi et al. (2014) and Vidal et al. (2014). The set of instances considers a combination of geographically distributed customers that are uniform and that are clustered. The number of customers in each instance varies between 50 and 360 . The number of depots per instance ranges from 2 to 9 . The set of instances are adapted from typical instances for the MDVRP, as presented in Salhi \& Sari (1997). In particular, the same locations for customers and depots as the same demands are considered. In addition, five types of vehicles $v_{k}$ are generated with the following characteristics: $Q_{k}=(0.4+0.2 k) Q^{*}, F_{k}=70+10 k$ and $\alpha_{k}=0.7+0.1 k$ with $k \in$ $\{1,2, \ldots, 5\} . Q^{*}$ refers to the vehicle capacity in the original instance.

The overall algorithm (AGM) is implemented in $\mathrm{C}++$, and the computational experiments are performed on an Intel Core Duo CPU (2.7 GHz) under Windows 7 with 4 GB of memory. The ILP models are optimally solved using the ILP solver CPLEX 12.5. The proposed algorithm is compared (see Tables 13) with the most effective published heuristics proposed for the MDHFVRP: SS97 of Salhi \& Sari (1997), SIW13, VNS1 and VNS2 of Salhi, Imran, \& Wassan (2014), and HGSADC of Vidal, Crainic, Gendreau, \& Prins (2014).

\subsection{Comparison of $S S 97$ and $A G M$}

Table 1 presents the results of the comparison between the methodology SS9 by Salhi \& Sari (1997) and AGM on 14 benchmarking instances proposed in the literature. The first three columns correspond to the characteristics of the instances: the number of the instance, the number of customers $(n)$ and the number 
of depots of each instance $(m)$. The next two columns show the results obtained for each of the proposed methodologies. The final column shows the gap of the solution of AGM with respect to the proposed approach SS97. A negative value indicates that the solution found by the AGM is better than the solution reported by SS97, and a positive value indicates otherwise.

The results show that AGM outperforms the performance of the algorithm SS97. Indeed, algorithm AGM obtains better results on 11 instances over 14 instances for the MDHFVRP. The three remaining instances obtain a gap of less than $2.2 \%$.

Table 1

Comparison of the algorithms SS97 and AGM

\begin{tabular}{cccccc}
\hline Instance & $\boldsymbol{n}$ & $\boldsymbol{m}$ & $\boldsymbol{S S 9 7}$ & AGM & Gap (\%) \\
\hline 1 & 50 & 4 & 1526,7 & 1513,12 & $-0,8895$ \\
2 & 50 & 4 & 992,8 & 981,809 & $-1,1071$ \\
3 & 75 & 2 & 1611,1 & 1605,77 & $-0,3308$ \\
4 & 100 & 2 & 2361,9 & 2338,43 & $-0,9937$ \\
5 & 100 & 2 & 1498,4 & 1491,83 & $-0,0044$ \\
6 & 100 & 3 & 2277,5 & 2252,54 & $-1,0959$ \\
7 & 100 & 4 & 2297,1 & 2294,79 & $-0,1006$ \\
8 & 80 & 2 & 2108,2 & 2079,98 & $-1,3386$ \\
9 & 80 & 2 & 2126,8 & 2116,78 & $-0,4711$ \\
10 & 80 & 2 & 2160,12 & 2160,46 & $+0,0157$ \\
11 & 160 & 4 & 4116,2 & 4056,93 & $-1,4399$ \\
12 & 240 & 6 & 6217 & 6193,38 & $-0,3799$ \\
13 & 249 & 3 & 6211,4 & 6347,27 & $+2,1874$ \\
14 & 360 & 9 & 9184,6 & 9353,0 & $+1,8335$ \\
\hline
\end{tabular}

\subsection{Comparison of SIW13, VNS1 and VNS2 algorithms and AGM}

Table 2 shows the comparison of the obtained results with the SIW1, VNS1 and VNS2 approaches proposed by Salhi, Imran, \& Wassan (2014) on 14 benchmarking instances. Note that our proposed approach obtains better solutions than those reported by the SIW1, VNS1 and VNS2 algorithms on 4 out of 14 instances. For all other instances, the gap ranges from $0.25 \%$ and $4.7 \%$.

Table 2

Comparison of the SIW13, VNS1, VNS2 and AGM algorithms

\begin{tabular}{ccccccc}
\hline Instance & $\boldsymbol{n}$ & $\boldsymbol{m}$ & $\begin{array}{c}\boldsymbol{S I W 1 3} \\
\boldsymbol{V N \boldsymbol { N } 1}\end{array}$ & $\begin{array}{c}\boldsymbol{S} \boldsymbol{S \boldsymbol { W 1 3 }} \\
\boldsymbol{V N \boldsymbol { S } 2}\end{array}$ & AGM & Gap (\%) \\
\hline 1 & 50 & 4 & 1503,3 & 1499,3 & 1513,12 & $+0,6532$ \\
2 & 50 & 4 & 974,9 & 984,5 & 981,809 & $-0,2733$ \\
3 & 75 & 2 & 1571,2 & 1588,4 & 1605,77 & $+1,0935$ \\
4 & 100 & 2 & 2321,9 & 2313,7 & 2338,43 & $+0,7119$ \\
5 & 100 & 2 & 1459,3 & 1466,9 & 1491,83 & $+1,6995$ \\
6 & 100 & 3 & 2224,0 & 2246,9 & 2252,54 & $+0,2510$ \\
7 & 100 & 4 & 2249,2 & 2256,4 & 2294,79 & $+1,7014$ \\
8 & 80 & 2 & 2085,8 & 2076,2 & 2079,98 & $-0,2790$ \\
9 & 80 & 2 & 2118,6 & 2096,4 & 2116,78 & $-0,0859$ \\
10 & 80 & 2 & 2188,6 & 2193,3 & 2160,46 & $-1,2857$ \\
11 & 160 & 4 & 4001,8 & 4024,9 & 4056,93 & $+0,7958$ \\
12 & 240 & 6 & 5994,6 & 5970,5 & 6193,38 & $+3,3159$ \\
13 & 249 & 3 & 6094,7 & 6068,8 & 6347,27 & $+4,1441$ \\
14 & 360 & 9 & 8935,6 & 8883,1 & 9353,0 & $+4,6712$ \\
\hline
\end{tabular}




\subsection{Comparison of HGSADC and AGM}

Table 3 presents the comparison between the HGSADC methodology by Vidal, Crainic, Gendreau, \& Prins (2014) and AGM on 14 instances of the proposed literature. Table 3 shows that the proposed approach obtains solutions close to those reported by the HGSADC algorithm. Indeed, the gap ranges between $0.0157 \%$ and $7.4 \%$.

Table 3

Comparison of HGSADC and AGM algorithms

\begin{tabular}{cccccc}
\hline Instance & $\boldsymbol{n}$ & $\boldsymbol{m}$ & HGSAD & AGM & Gap (\%) \\
\hline 1 & 50 & 4 & 1477,73 & 1513,12 & 2,3949 \\
2 & 50 & 4 & 957,73 & 981,809 & 2,5142 \\
3 & 75 & 2 & 1569,67 & 1605,77 & 2,2998 \\
4 & 100 & 2 & 2292,64 & 2338,43 & 1,9973 \\
5 & 100 & 2 & 1453,64 & 1491,83 & 2,6272 \\
6 & 100 & 3 & 2208,66 & 2252,54 & 1,9867 \\
7 & 100 & 4 & 2198,91 & 2294,79 & 4,3603 \\
8 & 80 & 2 & 2072,18 & 2079,98 & 0,3764 \\
9 & 80 & 2 & 2096,39 & 2116,78 & 0,9726 \\
10 & 80 & 2 & 2160,12 & 2160,46 & 0,0157 \\
11 & 160 & 4 & 3973,47 & 4056,93 & 2,1004 \\
12 & 240 & 6 & 5887,43 & 6193,38 & 5,1967 \\
13 & 249 & 3 & 5998,7 & 6347,27 & 6,0538 \\
14 & 360 & 9 & 8709,26 & 9353,0 & 7,3914 \\
\hline
\end{tabular}

\section{Concluding remarks}

We have proposed an effective metaheuristic algorithm for the Multi-Depot Vehicle Routing Problem with a Heterogeneous Fleet (MDHFVRP). The MDHFVRP considered a fixed cost related to the used vehicles and a variable cost associated with each type of vehicle. The former algorithm combines genetic operators with local search strategies to explore the solution space to find high-quality solutions to the MDHFVRP. A hybrid initialization procedure that provides a good level of diversity for the initial population was presented. The proposed approach combines heuristics for populations based on permutations together with a splitting process adapted from the classic VRP. Different strategies of interand intra-route neighborhoods enable an intensification of the search space, thereby allowing different feasible regions of the highest quality to be explored for each generation of the proposed methodology.

We have compared the proposed algorithm with the most effective published heuristics for the MDHFVRP on a set of benchmark instances from the literature. The results have demonstrated the effectiveness of the proposed algorithm. The gaps obtained with respect to the best reported results demonstrate the remarkable contribution of the former algorithm for future research. The results obtained suggest that the proposed framework could be applied to other problems with similar characteristics to the MDHFVRP such as the location-routing problem with a heterogeneous fleet, the Multi-Depot Vehicle Routing Problem (MDVRP) and the Periodic location routing problem (PLRP), among others. 


\section{References}

Aras, N., Aksen, D., \& Tekin, M. T. (2011). Selective multi-depot vehicle routing problem with pricing. Transportation Research Part C, 19(5), 866-884.

Baldacci, R., \& Dell'Amico, M. (2010). Heuristic algorithms for the multi-depot ring-star problem. European Journal of Operational Research, 203(1), 270-281.

Bettinelli, A., Ceselli, A., \& Righini, G. (2011). A branch-and-cut-and-price algorithm for the multidepot heterogeneous vehicle routing problem with time windows. Transportation Research Part C, 19(5), 723-740.

Bolaños, R., Echeverry, M., \& Escobar, J. (2015). A multiobjective non-dominated sorting genetic algorithm (NSGA-II) for the Multiple Traveling Salesman Problem. Decision Science Letters, 4(4), 559-568.

Braekers, K., Ramaekers, K., \& Van Nieuwenhuyse, I. (2016). The vehicle routing problem: State of the art classification and review. Computers \& Industrial Engineering, 99, 300-313.

Cassidy, P. J., \& Bennett, H. S. (1972). A Multi-Depot Vehicle Scheduling System. Operational Research Quarterly, 23(2), 151-163.

Clarke, G., \& Wright, J. (1964). Scheduling of vehicles from a central depot to a number of delivery point. Operation Research, 12(4), 568-581.

Crevier, B., Cordeau, J.-F., \& Laporte, G. (2007). The multi-depot vehicle routing problem with interdepot routes. European Journal of Operational Research, 176(2), 756-773.

Dantzig, G., \& Ramser, J. (1959). The truck dispatching problem. Management Science, 6(1), 80-91.

Escobar, J.W., Linfati, R., \& Toth, P. (2013). A two-phase hybrid heuristic algorithm for the capacitated location-routing problem. Computers \& Operations Research, 40(1), 70-79.

Escobar, J.W., Linfati, R., Toth, P., \& Baldoquin, M. G. (2014). A hybrid granular tabu search algorithm for the multi-depot vehicle routing problem. Journal of Heuristics, 20(5), 483-509.

Escobar, J.W., Linfati, R., Baldoquin, M. G., \& Toth, P. (2014). A Granular Variable Tabu Neighborhood Search for the capacitated location-routing problem. Transportation Research Part B: Methodological, 67, 344-356.

Escobar, J.W., Linfati, R., \& Adarme-Jaimes, W. (2015). A hybrid metaheuristic algorithm for the capacitated location routing problem. Dyna, 82(189), 243-251.

Goldberg, D. E., \& Lingle, R. L. (1985). The traveling salesman problem. Proc. First Int. Conf. Genetic Algorithms and their Applications, 154-159.

Gulczynski, D., Golden, B., \& Wasil, E. (2011). The multi-depot split delivery vehicle routing problem: An integer programming-based heuristic, new test problems, and computational results. Computers \& Industrial Engineering, 61(3), 794-804.

Gutin, G., Yeo, A., \& Zverovich, A. (2002). Traveling salesman should not be greedy: domination analysis of greedy-type heuristics for the TSP. Proceedings of the romanian academy, series $\mathrm{A}, 117$, 81-86.

Ho, W., Ho, G. T., Jib, P., \& Laub, H. W. (2008). A hybrid genetic algorithm for the multi-depot vehicle routing problem. Engineering Applications of Artificial Intelligence, 21(4), 548-557.

Holland, J. H. (1975). Adaptation in Natural and Artificial Systems. Ann Arbor, Michigan, USA: The University of Michigan Press.

Karakatič, S., \& Podgorelec, V. (2015). A survey of genetic algorithms for solving multi depot vehicle routing problem. Applied Soft Computing, 27, 519-532.

Kuo, Y., \& Wang, C.-C. (2012). A variable neighborhood search for the multi-depot vehicle routing problem with loading cost. Expert Systems with Applications, 39(8), 6949-6954.

Lin, S., \& Kernighan, B. W. (1973). An effective heuristic algorithm for the traveling-salesman problem. Operations Research, 21(2), 498-516.

Linfati, R., Escobar, J. W., \& Gatica, G. (2014). Un algoritmo metaheurístico para el problema de localización y ruteo con flota heterogénea. Ingeniería y Ciencia, 10(19), 55-76. 
Mancini, S. (2016). A real-life multi depot multi period vehicle routing problem with a heterogeneous fleet: formulation and adaptive large neighborhood search based matheuristic. Transportation Research Part C: Emerging Technologies, 70, 100-112.

Mirabi, M., Ghomi, S. F., \& Jolai, F. (2010). Efficient stochastic hybrid heuristics for the multi-depot vehicle routing problem. Robotics and Computer-Integrated Manufacturing, 26(6), 564-569.

Nagy, G., \& Salhi, S. (2005). Heuristic algorithms for single and multiple depot vehicle routing problems with pickups and deliveries. European Journal of Operational Research, 162(1), 126-141.

Oliver, I. M., Smith, D. J., \& Holland, J. (1987). A study of permutation crossover operators on the traveling salesman problem. Proc. Second Int. Conf. Genetic Algorithms and their Applications, 224230.

Prins, C. (2009). Two memetic algorithms for heterogeneous fleet vehicle routing problem. Engineering Applications of Artificial Intelligence, 22(6), 916-928.

Renaud, J., Laporte, G., \& Boctor, F. F. (1996). A Tabu Search Heuristic For The Multi-Depot Vehicle Routing Problem. Computers Operations Researchs, 23(3), 229-235.

Salhi, S., \& Sari, M. (1997). A multi-level composite heuristic for the multi-depot vehicle fleet mix problem. European Journal of Operational Research, 103(1), 95-112.

Salhi, S., Imran, A., \& Wassan, N. A. (2014). The multi-depot vehicle routing problem with heterogeneous vehicle fleet: Formulation and a variable neighborhood search implementation. Computers \& Operations Research, 52, 315-32.

Subramanian, A., Vaz-Penna, P. H., Uchoa, E., \& Ochi, L. S. (2012). European Journal of Operational Research. A hybrid algorithm for the Heterogeneous Fleet Vehicle Routing Problem, 221(2), 285-295.

Syswerda, G. (1991). Schedule optimization using genetic algorithms. In A Handbook of Genetic Algorithms (Edited by L. Davis), 332-349.

Toth, P., \& Vigo, D. (2002). An overview of vehicle routing problems. In P. Toth, D. Vigo, \& M. o. Applications (Ed.), The Vehicle Routing Problem (pp. 1-26). SIAM.

Vidal, T., Crainic, T. G., Gendreau, M., \& Prins, C. (2014). Implicit depot assignments and rotations in vehicle routing heuristics. European Journal of Operational Research, 237, 15-28.

Wren, A., \& Holliday, A. (1972). Computer Scheduling of Vehicles from One or More Depots to a Number of Delivery Points. Operational Research Quarterly, 23(3), 333-344.

Wu, T.-H., Low, C., \& Bai, J.-W. (2002). Heuristic solutions to multi-depot location-routing problems. Computers \& Operations Research, 29(10), 1393-1415.

Xu, Y., Wang, L., \& Yang, Y. (2012). A new variable neighborhood search algorithm for the multi depot heterogeneous vehicle routing problem with time windows. Electronic Notes in Discrete Mathematics, 39, 289-296.

Yücenur, G. N., \& Demirel, N. C. (2011). A new geometric shape-based genetic clustering algorithm for the multi-depot vehicle routing problem. Expert Systems with Applications, 38(9), 11859-11865.

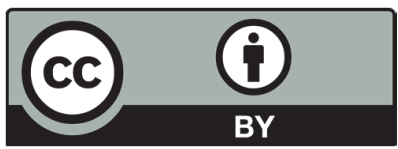

(C) 2018 by the authors; licensee Growing Science, Canada. This is an open access article distributed under the terms and conditions of the Creative Commons Attribution (CCBY) license (http://creativecommons.org/licenses/by/4.0/). 